\title{
Mechanical ventilation strategy for pulmonary rehabilitation based on patient-ventilator interaction
}

\author{
HAO LiMing ${ }^{1 \dagger}$, LI Xiao ${ }^{2 \dagger}$, SHI Yan ${ }^{1}$, CAI MaoLin ${ }^{1}$, REN Shuai ${ }^{1,6^{*}}$, XIE Fei $^{3}$, LI YaNa ${ }^{2}$, \\ WANG $\mathrm{Na}^{1 \dagger}$, WANG YiXuan ${ }^{1}$, LUO ZuJin ${ }^{4} \&$ XU Meng ${ }^{5 *}$ \\ ${ }^{1}$ School of Automation Science and Electrical Engineering, Beihang University, Beijing 100191, China; \\ ${ }^{2}$ Department of Rehabilitation, The Fouth Medical Center of PLA General Hospital, Beijing 100048, China; \\ ${ }^{3}$ Department of Pulmonary and Critical Care Medicine, Chinese PLA General Hospital, Beijing 100039, China; \\ ${ }^{4}$ Department of Respiratory and Critical Care Medicine, Beijing Engineering Research Center of Respiratory and Critical Care Medicine, \\ Beijing Institute of Respiratory Medicine, Beijing Chao-Yang Hospital, Capital Medical University, Beijing 100043, China; \\ ${ }^{5}$ Department of Orthopedics, Chinese PLA General Hospital, Beijing 100039, China; \\ ${ }^{6}$ State Key Laboratory of Fluid Power and Mechatronic Systems, Zhejiang University, Hangzhou 310027, China
}

Received September 2, 2020; accepted January 11, 2021; published online February 10, 2021

\begin{abstract}
Mechanical ventilation is an effective medical means in the treatment of patients with critically ill, COVID-19 and other pulmonary diseases. During the mechanical ventilation and the weaning process, the conduct of pulmonary rehabilitation is essential for the patients to improve the spontaneous breathing ability and to avoid the weakness of respiratory muscles and other pulmonary functional trauma. However, inappropriate mechanical ventilation strategies for pulmonary rehabilitation often result in weaning difficulties and other ventilator complications. In this article, the mechanical ventilation strategies for pulmonary rehabilitation are studied based on the analysis of patient-ventilator interaction. A pneumatic model of the mechanical ventilation system is established to determine the mathematical relationship among the pressure, the volumetric flow, and the tidal volume. Each ventilation cycle is divided into four phases according to the different respiratory characteristics of patients, namely, the triggering phase, the inhalation phase, the switching phase, and the exhalation phase. The control parameters of the ventilator are adjusted by analyzing the interaction between the patient and the ventilator at different phases. A novel fuzzy control method of the ventilator support pressure is proposed in the pressure support ventilation mode. According to the fuzzy rules in this research, the plateau pressure can be obtained by the trigger sensitivity and the patient's inspiratory effort. An experiment prototype of the ventilator is established to verify the accuracy of the pneumatic model and the validity of the mechanical ventilation strategies proposed in this article. In addition, through the discussion of the patient-ventilator asynchrony, the strategies for mechanical ventilation can be adjusted accordingly. The results of this research are meaningful for the clinical operation of mechanical ventilation. Besides, these results provide a theoretical basis for the future research on the intelligent control of ventilator and the automation of weaning process.
\end{abstract}

mechanical ventilation, pulmonary rehabilitation, phases division, fuzzy control, patient-ventilator interaction

Citation: Hao L M, Li X, Shi Y, et al. Mechanical ventilation strategy for pulmonary rehabilitation based on patient-ventilator interaction. Sci China Tech Sci, 2021, 64: 869-878, https://doi.org/10.1007/s11431-020-1778-8

$\dagger$ These authors contributed equally to this work.

*Corresponding authors (email: renshuai@buaa.edu.cn; drxm301@163.com) 


\section{Introduction}

Mechanical ventilation is commonly used in the treatment of critically ill patients. According to the reports, over $40 \%$ of the patients in ICU need to be treated with the support of mechanical ventilation $[1,2]$. In addition, the patients with pulmonary diseases, such as chronic obstructive pulmonary disease (COPD), also need ventilators to help them breathe smoothly. Moreover, COPD has become one of the most common causes of death in the world [3-5]. In addition, pneumonia caused by the virus presents great challenges with respiratory treatment. As of July 15th, 2020, more than 13.24 million people had been infected with COVID-19, and there are more than 4.98 million confirmed cases in real time. In the face of this unprecedented epidemic, mechanical ventilation is still the most effective way to maintain the lives of patients [6]. Therefore, it is urgent to solve the clinical problems in mechanical ventilation.

During the process of mechanical ventilation, the ventilator supplies the air with a certain pressure or flow supporting the patient's breathing. Mechanical ventilation needs to be operated at a certain intensity and in the limited time to maintain patient's life. The prolonged ventilation usually leads to the weakness of respiratory muscles, which is harmful to the improvement of the patient's spontaneous breathing ability, and even weakens the patient's respiratory function [7-10]. Hence, the patients need to gradually get rid of their dependence on the ventilator under appropriate conditions. Medically, the process of removing a patient form the ventilator is defined as weaning [11]. The delay in weaning often results in diaphragm failure, nosocomial infection, pulmonary functional trauma and even the death of life [12-16]. It is important to complete the weaning in time, and the training of the patient's spontaneous breathing ability during the weaning process is helpful for the smooth weaning [17]. This involves the pulmonary rehabilitation during mechanical ventilation. Different from the pulmonary rehabilitation in terms of physical exercise and psychological guidance for patients, the pulmonary rehabilitation in this paper refers to the maintenance and gradual recovery of the patient's spontaneous breathing ability through the adjustment of the ventilator's controlling parameters during mechanical ventilation.

The previous research in a review has summarized the clinical method used to train the inspiratory muscle. For example, lighter sedation protocols can be applied for the patients in mechanical ventilation to activate the diaphragm [18]. But this method is not effective enough to exercise the patient's respiratory muscle thoroughly. Clinically, there are some commonly used methods to exercise patient's spontaneous breathing ability, such as inspiratory resistive training, threshold pressure training, and adjusting triggering sensitivity [19-21]. During the period of weaning from mechan- ical ventilation, there are several ways to undertake inspiratory muscle training, such as isocapnic/normocapnic hyperpnoea training, the application of devices that impose resistive, or threshold loads [22]. Some literature also provides survey reports on the effects of inspiratory muscle training [23-25]. However, these strategies focus on improving the state of the respiratory muscles, without making adjustments to the control parameters of mechanical ventilation, and lacking a more comprehensive improvement of the effect of ventilation therapy.

In addition, with the development of artificial intelligence, some novel computer technology has also been applied to the pulmonary rehabilitation and the weaning process. Therefore, some novel mechanical ventilation strategies have been proposed. Prasad et al. [26] used the reinforcement learning method to learn a simple ventilator weaning policy from the clinical examples. They have proposed a data-driven approach to optimize the weaning process of the patients in ICU. This method is novel and effective, but it requires a mass of data as the basis for machine learning, which increases the difficulty of its clinical operation.

In this paper, the mechanical ventilation strategy for pulmonary rehabilitation in mechanically ventilated patients is studied from the perspective of patient-ventilator interaction. The process of pulmonary rehabilitation in mechanical ventilation aims to train and improve the patient's spontaneous breathing ability, so as to prevent the decline of respiratory function caused by prolonged dependence on the ventilator. However, the improvement of the patient's selfbreathing ability must be confronted with the ventilator to some extent. Therefore, the ventilator parameters need to be adjusted constantly to accommodate the patient's spontaneous breathing while encouraging the patient to breathe autonomously. The past research results have been carried out in terms of clinical dosage and clinical data mining [2729]. The research in this paper is based on the pneumatic model of patient-ventilator interaction. The ventilator works in a pressure support ventilation mode (PSV). And each breathing cycle is divided into four different phases. According to the patient-ventilator interaction phenomenon in different phases, the mechanical ventilation strategy for pulmonary rehabilitation is adjusted. The results of this paper are the basis of the research on fuzzy adaptive control in mechanical ventilation and personalized ventilation strategy in the future studies.

\section{Materials and methods}

\subsection{Pneumatic model of patient-ventilator interaction}

The mechanical ventilation system can be regarded as the interaction process between the patient and the ventilator. In the previous research, we had studied the modelling of the 
mechanical ventilation system [30-33]. The interaction relationship between the patient and the ventilator can also be studied as a pneumatic model. This model consists of two connected pneumatic systems, namely the ventilator pneumatic system and the patient pneumatic system. As shown in Figure 1, the former is made up of the fan that provides the air, the pressure and flow sensors that monitor the air supply status, the signal acquisition and the control centre. The latter is made up of the vacuum pump corresponding to the diaphragm, the elastic container corresponding to the lungs and the throttle. The vacuum pump provided the power for spontaneous breathing. The elastic container can reflect the compliance characteristic of the lungs. The cross-sectional area of the throttle reflects the air resistance characteristic of the respiratory system. The ventilator pneumatic system and the patient pneumatic system are connected by a tube, whose compliance and resistance can be ignored under ideal conditions.

The patient's spontaneous breathing is driven by the effort of the diaphragm and other respiratory muscles, such as intercostal muscles [34-36]. This effort can produce negative pressure inside the chest, which triggers the ventilator to work. The change of pressure inside the thorax caused by the effort of respiratory muscles is represented as $P_{\text {muscle. This }}$ change in pressure produces a change in the volume of lungs. According to the ideal gas state equation $(P V=m R T)$ [37], the volume change results in another change of pressure, which is represented as $P_{\mathrm{L}}$. The equilibrium state of the thoracic environment is set as atmospheric pressure, $P_{\mathrm{ATM}}$. $P_{\text {muscle }}$ and $P_{\mathrm{L}}$ together affect intrapulmonary pressure, $P_{\text {chest }}$. The ventilator operates in PSV mode. The difference between $P_{\text {chest }}$ and $P_{\mathrm{V}}$ produces the airflow in the airway and the ventilation tube. Inhale $\left(Q_{\text {in }}\right)$ when $P_{\mathrm{V}}$ is greater than $P_{\text {chest }}$ and exhale $\left(Q_{\text {out }}\right)$ when $P_{\text {chest }}$ is greater than $P_{\mathrm{V}}$. The pressure in the airway is represented as $P_{\mathrm{AW}}$. The relationship among these pressure parameters is shown in Figure 2.

According to the diagram above, the mathematical formula can be derived as the following. And based on the ideal gas state equation, the differential relationship between pressure and volume or volumetric flow can be further derived. The derivation principle has been explained in previous studies and will not be elaborated here [30,38-41]. The mathematical model is illustrated in the following equations.

(1) Intrapulmonary pressure

$P_{\text {chest }}=P_{\mathrm{L}}+P_{\text {muscle }}-P_{\text {ATM }}$

(2) Pressure integral equation under volume influence

$P_{\mathrm{L}}=\int \frac{\rho R T Q V}{V^{2}+C m R T} \mathrm{~d} t$.

(3) Volumetric flow equation

$Q_{\text {in }}=-Q_{\text {out }}=\frac{A_{\mathrm{e}} P_{\text {up }}}{\rho \sqrt{R T}} \sqrt{1-r-\frac{\left(\frac{P_{\text {down }}}{P_{\text {up }}}-r\right)^{2}}{1-r}}$.

When $P_{\mathrm{V}}$ is greater than $P_{\text {chest }}$, the patient inhales. At this time, $P_{\text {up }}$ is $P_{\mathrm{V}}$ and $P_{\text {down }}$ is $P_{\text {chest }}$. In contrast, the patent exhales, and $P_{\text {up }}$ is equal to $P_{\text {chest }}$. In the formula, $\rho$ is the air density $\left(\mathrm{kg} / \mathrm{m}^{3}\right)$, and $r$ is equal to 0.528 , which is the critical pressure ratio.

(4) Respiratory mechanical parameters

Compliance $(C)$ and resistance $\left(R_{\mathrm{r}}\right)$ are the two most important respiratory mechanical parameters. The compliance can reflect the interaction between the pressure and the volume in the lung cavity (as shown in eq. (4)). In the pneumatic model, the air resistance is inversely correlated with the cross-sectional area of the throttle $\left(A_{\mathrm{e}}\right)$. The influence of

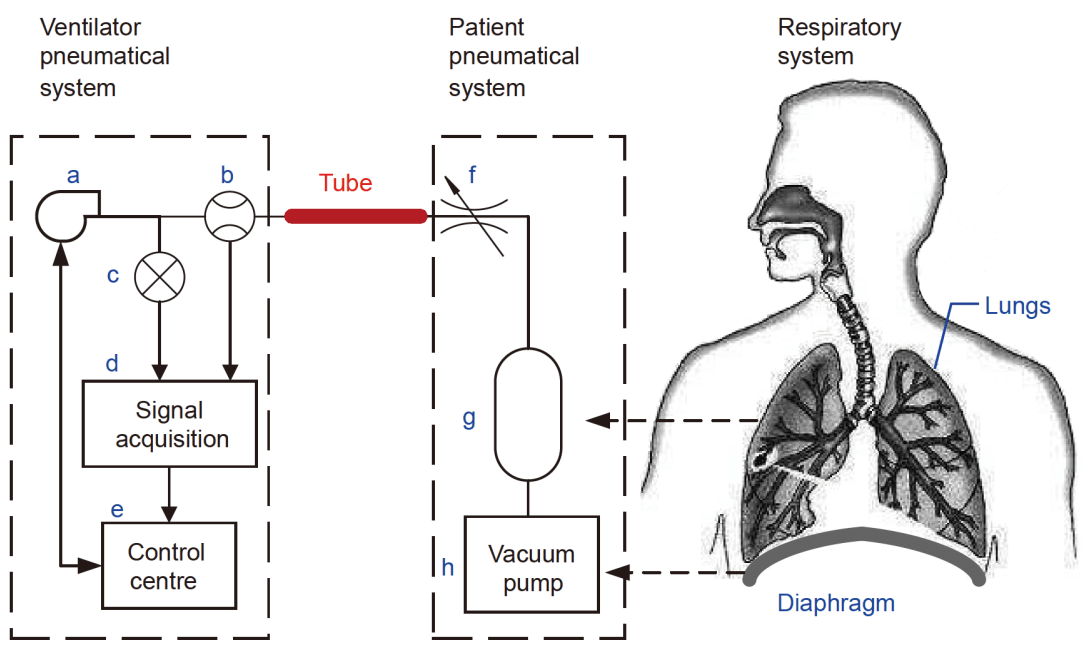

Figure 1 (Color online) Schematic diagram of the pneumatic model of the patient-ventilator interaction. The ventilator pneumatic system: (a) fan; (b) flow sensor; (c) pressure sensor; (d) signal acquisition module; (e) control centre. The patient pneumatic system: (f) throttle; (g) elastic container; (h) vacuum pump. 


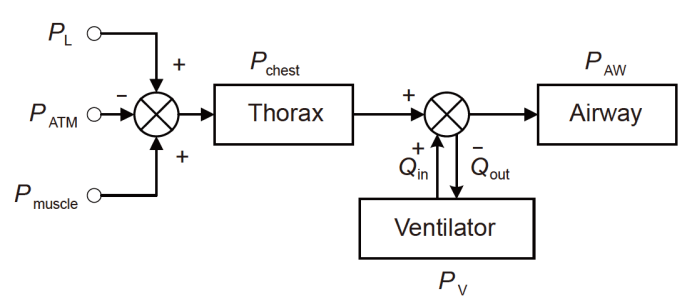

Figure 2 Mathematical diagram of a mechanical ventilation system. The airflow of the ventilator is affected by the pressure difference between the thorax and the airway.

air resistance on ventilation flow can be reflected in eq. (3).

$C=\frac{\mathrm{d} P_{L}}{\mathrm{~d} V}$

According to the equations above, the pressure waveform of the mechanical ventilation system can be obtained. As shown in Figure 3, the action of $P_{\text {muscle }}$ causes the initial negative pressure of $P_{\text {chest }} . P_{\mathrm{L}}$ satisfies the ideal gas equation with the lung volume during respiration. $P_{\mathrm{AW}}$ can be measured directly by sensors and other external devices. The pressure relationship in this part lays a foundation for the identification of respiratory mechanics parameters.

\subsection{Pulmonary rehabilitation strategies at different phases of each breathing cycle}

During the mechanical ventilation in PSV mode, each breathing cycle can be divided into four phases based on the respiratory properties. As shown in Figure 4, these four divisions respectively are: (1) triggering phase; (2) inhalation phase; (3) switching phase; (4) exhalation phase. The trigger phase corresponds to the process in which the patient's spontaneous breathing triggers the ventilator to work. The inhalation phase corresponds to the process in which $P_{\mathrm{AW}}$ rises to the plateau pressure while maintaining pressure support. The switch phase corresponds to the transition between inhalation and exhalation, and determines the patient's $I / E$ ratio. The exhalation phase corresponds to the patient's exhalation and usually presents the value of positive endexpiratory pressure (PEEP).

In the process of pulmonary rehabilitation, there are different parameter values corresponding to different ventilation stages, and different ventilation strategies should be adopted.

\subsubsection{Triggering phase}

To reflect the patient's inspiratory effort, a novel variable, $\mathrm{PT}$, is defined in this article. The mathematical expression of PT is shown in eq. (5). This variable is expressed in the respiratory waveform as the area of the part enclosed by the pressure baseline and the negative pressure at the beginning of ventilation. This part is shown as the the shaded area in

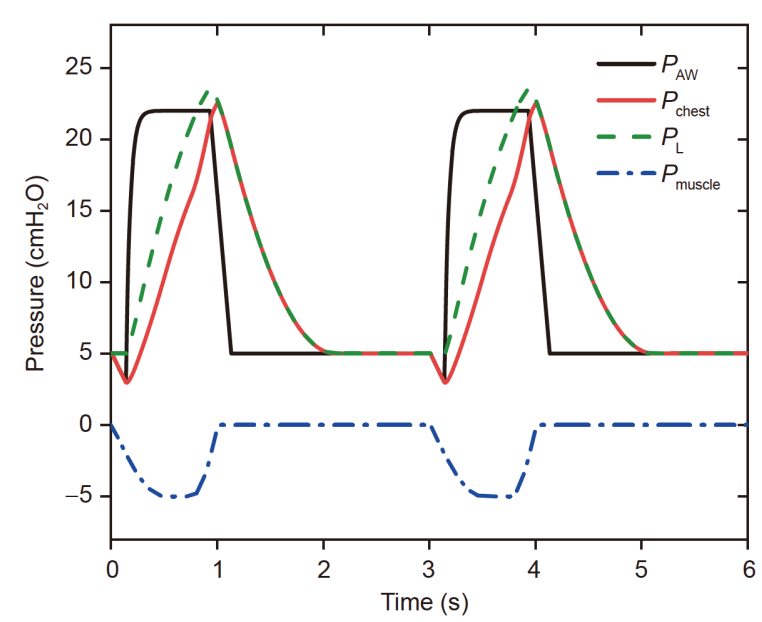

Figure 3 (Color online) Pressure waveform of the mechanical ventilation system. The four curves with different line type represent the air pressure in different parts of the ventilation system.

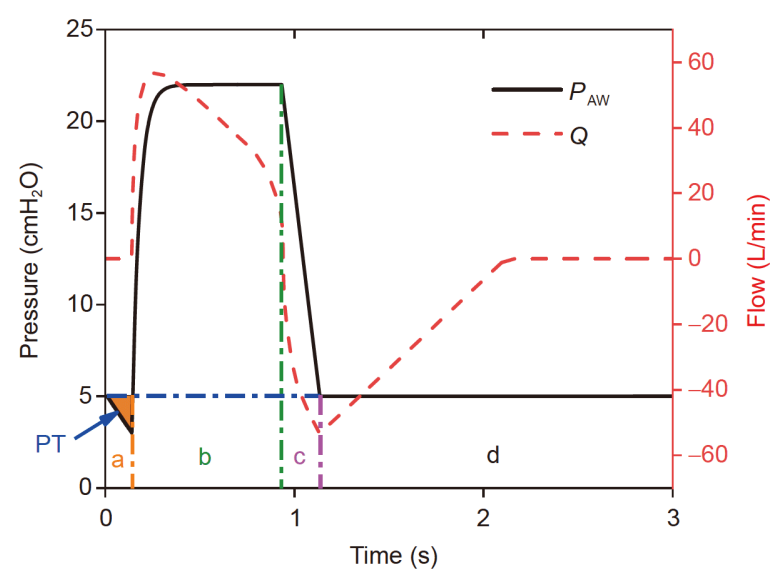

Figure 4 (Color online) The phases of a single ventilation period. Each breathing cycle is divided into four different periods, namely, (a) triggering phase; (b) inhalation phase; (c) switching phase; (d) exhalation phase.

Figure 4. As the integration of $P_{\mathrm{AW}}$ and time, the PT's unit is expressed as " $\mathrm{cmH}_{2} \mathrm{O} \cdot \mathrm{s}$ ". The physical meaning of this variable is the patient's effort to produce negative pressure while inhales in the trigger phase. By adjusting the trigger sensitivity, the value of PT varied with the intensity of the patient's spontaneous breathing. The higher value of PT indicates the stronger the ability of the patient to breathe autonomously.

$\mathrm{PT}=\int_{0}^{t_{a}} P_{\mathrm{AW}} \mathrm{d} t$.

Figure 5 shows the pressure waveforms at different levels of pressure support and spontaneous breathing intensity. The part enclosed by the dotted line reflects different PT values, representing different breathing intensities of the patient. The process of pulmonary rehabilitation needs to gradually reduce the trigger sensitivity, to increase the PT value, and to reduce the degree of pressure support in response. From 


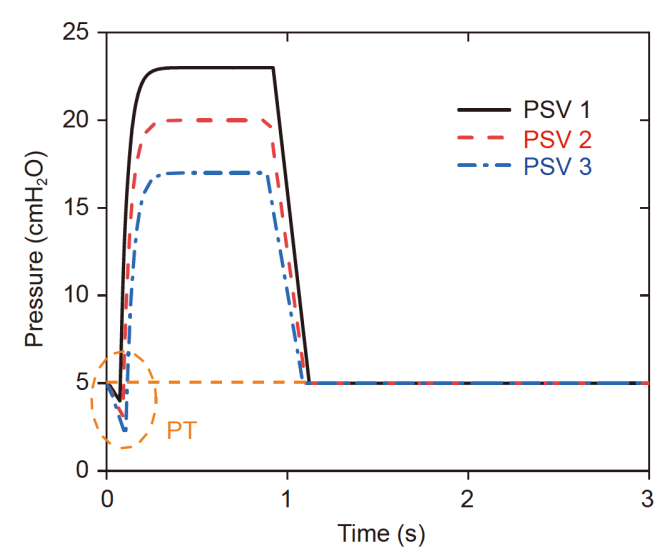

Figure 5 (Color online) Pressure waveforms in PSV mode at different levels of pressure support and spontaneous breathing intensity. The part marked by the dotted line reflects the inhalation effort in the triggering phase.

PSV1 to PSV3, the absolute value of the trigger pressure gradually increases, the corresponding PT value gradually increases, and the plateau pressure gradually decreases (as shown in Figure 5).

\subsubsection{Inhalation phase}

The inhalation phase corresponds to the increase in the support pressure of the ventilator and the maintenance of the plateau pressure. During the pulmonary rehabilitation, the plateau pressure of supporting pressure should be gradually reduced, corresponding to the improvement of the patient's spontaneous breathing ability and the decrease of trigger sensitivity. In this article, a novel respiratory pressure support strategy is proposed based on fuzzy logic for the first time. By establishing the fuzzy relationship among the value of PT, the trigger pressure $\left(P_{\text {tri }}\right)$ and the plateau pressure $\left(P_{\mathrm{S}}\right)$, the pressure support degree can be appropriately determined.

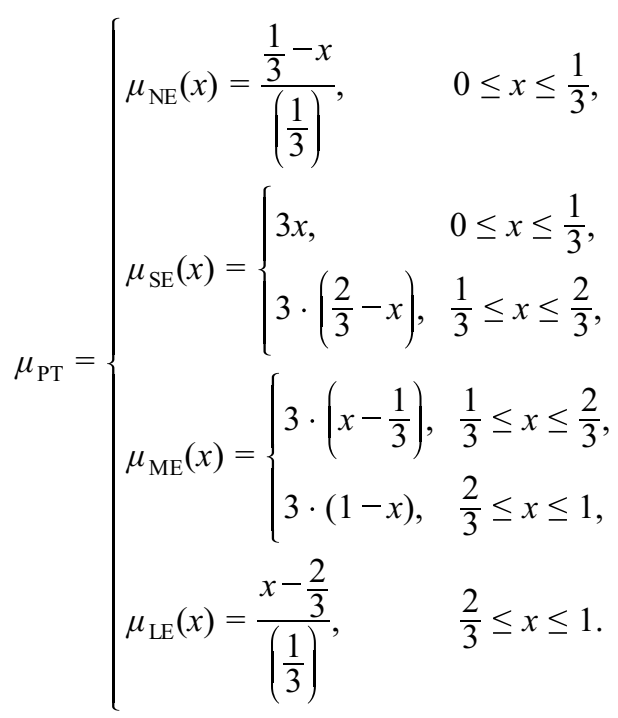

According to clinical experience, four critical values of PT can be determined, which are $0,1 / 3,2 / 3$ and $1\left(\mathrm{cmH}_{2} \mathrm{O} \cdot \mathrm{s}\right)$. The variable, PT, is divided into four fuzzy sets: NE (very little inhalation effort), SE (less inhalation effort), ME (medium inhalation effort), LE (large inhalation effort). The corresponding membership function is shown in eq. (6). The simulation results are shown in Figure 6.

Similarly, three critical values of the trigger pressure $\left(P_{\text {tri }}\right)$ are determined, namely 1,2 and $3\left(\mathrm{cmH}_{2} \mathrm{O}\right) . P_{\text {tri }}$ is divided into three fuzzy sets: HT (high trigger sensitivity), MT (moderate trigger sensitivity), LT (low trigger sensitivity). The corresponding membership function is shown in eq. (7).

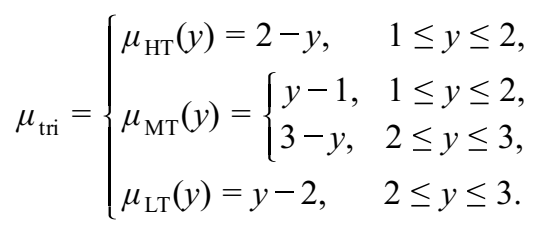

Based on the formula above, the membership function of the trigger pressure can be simulated as shown in Figure 7.

When the ventilator works in PSV mode, the plateau pressure $\left(P_{\mathrm{S}}\right)$ usually reflects the degree of pressure support. The higher the plateau pressure is, the stronger the support degree of the ventilator is. The plateau pressure is divided into five critical values of $12,14,16,18$, and $20\left(\mathrm{cmH}_{2} \mathrm{O}\right)$. According to the critical values, the plateau pressure is divided into 5 fuzzy sets, namely $P_{\mathrm{S}} 1, P_{\mathrm{S}} 2, P_{\mathrm{S}} 3, P_{\mathrm{S}} 4$ and $P_{\mathrm{S}} 5$, which represent five different pressure support levels. The corresponding membership function is shown in eq. (8). And the simulation results based on the equation are shown in Figure 8.

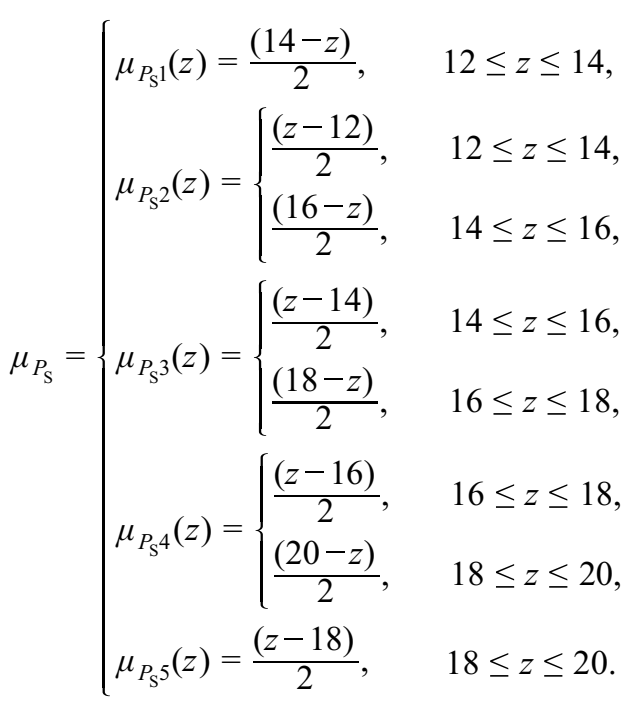

According to the clinical experience of mechanical ventilation during pulmonary rehabilitation, the fuzzy rules of ventilator's controlling strategy are designed. The fuzzy rules table is shown in Table 1. The fuzzy sets of $P_{\mathrm{S}}$ can be determined by the corresponding fuzzy sets of $P_{\text {tri }}$ and PT. 


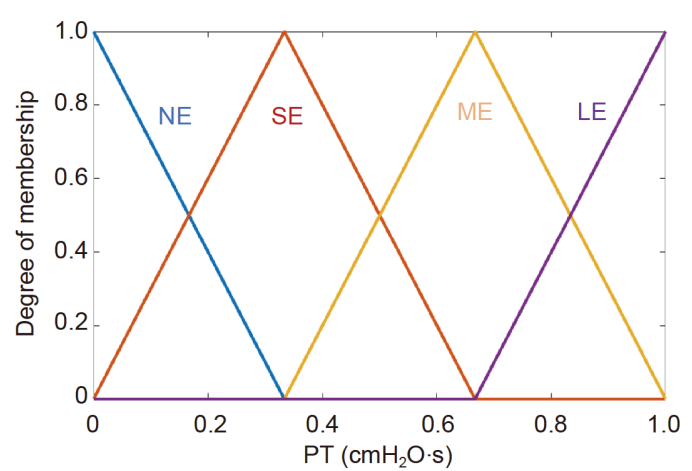

Figure 6 (Color online) Simulation of PT's membership function.

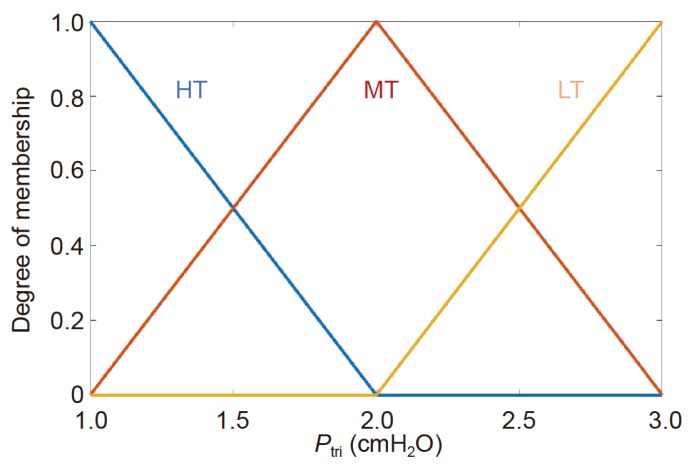

Figure 7 (Color online) Simulation of $P_{\text {tri }}$ 's membership function.

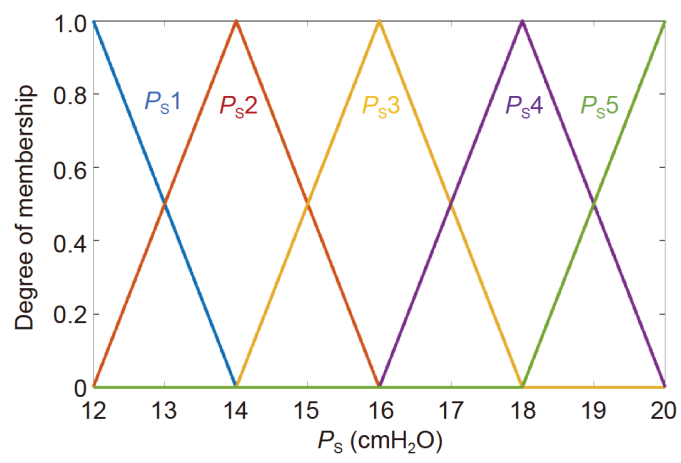

Figure 8 (Color online) Simulation of $P_{\mathrm{S}}$ 's membership function.

Table 1 Fuzzy rules table of the ventilator's controlling strategy

\begin{tabular}{lccccc}
\hline \multirow{2}{*}{ Plateau pressure $\left(P_{\mathrm{S}}\right)$} & \multicolumn{5}{c}{$\mathrm{PT}$} \\
\cline { 3 - 6 } & $\mathrm{NE}$ & $\mathrm{SE}$ & $\mathrm{ME}$ & $\mathrm{LE}$ \\
\hline \multirow{3}{*}{ Trigger pres- } & $\mathrm{HT}$ & $P_{\mathrm{S}} 5$ & $P_{\mathrm{S}} 4$ & $P_{\mathrm{S}} 4$ & $P_{\mathrm{S}} 5$ \\
sure $\left(P_{\text {tri }}\right)$ & $\mathrm{MT}$ & $P_{\mathrm{S}} 2$ & $P_{\mathrm{S}} 2$ & $P_{\mathrm{S}} 3$ & $P_{\mathrm{S}} 4$ \\
& $\mathrm{LT}$ & $P_{\mathrm{S}} 1$ & $P_{\mathrm{S}} 1$ & $P_{\mathrm{S}} 2$ & $P_{\mathrm{S}} 2$ \\
\hline
\end{tabular}

\subsubsection{Switching phase}

The switching of inhalation and exhalation in the process of mechanical ventilation depends on the peak volumetric flow
$\left(Q_{\text {peak }}\right)$ and the switching coefficient $(k)$. When the volumetric flow drops to $k \cdot Q_{\text {peak }}$, the support pressure of the ventilator starts to drop from the plateau pressure to the PEEP. The value of $k$ is usually $5 \%-10 \%$.

$k^{\prime}=\frac{\mathrm{PT}^{\prime}}{\mathrm{PT}_{0}} \cdot k_{0}$

When performing mechanical ventilation for pulmonary rehabilitation, as the patient's spontaneous breathing ability is strengthened, the patient's inspiration-to-expiration ratio changes, so that the switching coefficient $(k)$ can be increased appropriately. It is determined that the initial switching coefficient is $k_{0}$ and the corresponding PT value is $\mathrm{PT}_{0}$, then when the patient's $\mathrm{PT}$ value is $\mathrm{PT}^{\prime}$, the switching coefficient corresponds to $k^{\prime}$ (as shown in eq. (9)).

\subsubsection{Exhalation phase}

In the exhalation phase, in order to offset the impact of endogenous positive pressure, the ventilator is usually required to provide a certain pressure support, which is clinically called PEEP. In the process of pulmonary rehabilitation, as the patient's breathing function recovers, there is sufficient ability to expel excess gas from the chest cavity, so the support for PEEP can be gradually reduced. A relationship can be established between the adjustment of the PEEP and the trigger pressure. Determine that the initial PEEP is $\mathrm{PEEP}_{0}$, and the trigger sensitivity of the corresponding ventilation cycle is $P_{\text {trio. }}$. Then, when the trigger sensitivity is adjusted to $P_{\text {tri }}{ }^{\prime}$, try to adjust the PEEP to PEEP', as shown in

$\mathrm{PEEP}^{\prime}=\frac{P_{\text {tri0 }}}{P_{\text {tri }}{ }^{\prime}} \cdot \mathrm{PEEP}_{0}$.

Based on the control strategy of the mechanical ventilation for pulmonary rehabilitation, an experimental prototype of the ventilator is established in this research. The prototype consists of a power, a turbo fan, a motor driver, the control centre (STM32), a tube, a pressure and flow sensor, and a lung simulator (as shown in Figure 9). Besides, with a lung simulator (ASL5000), this experimental prototype can be used to verify the effectiveness of the ventilation strategies based on fuzzy logic and patient-ventilator interaction.

\section{Results and discussion}

The strategy of the mechanical ventilation for pulmonary rehabilitation proposed in this study is not invariable. In the clinical application process, appropriate adjustments should be made at any time according to the performance of the patient. During the process of pulmonary rehabilitation, there will be different degrees of patient-ventilator asynchrony due to the deviation of ventilator control parameters adjustment. Common phenomena fall into the following three situations. 


\subsection{Asynchrony phenomenon of ineffective triggering}

When the trigger sensitivity adjustment method is used for pulmonary rehabilitation training, if the trigger sensitivity is set too low, the patient's spontaneous breathing ability is insufficient, and sufficient negative pressure cannot be generated. At this time, an ineffective triggering phenomenon usually occurs. As shown in Figure 10, the solid line represents the pressure waveform of ineffective triggering, and the dotted line represents the normal triggering. Through the detection of the plateau pressure in each cycle, the ineffective triggering can be effectively measured.

The trigger sensitivity should be appropriately increased as the occurrence of the ineffective triggering. According to the control method based on fuzzy logic described above, a reasonable degree of pressure support $\left(P_{\mathrm{S}}\right)$ can be determined by the adjusted trigger pressure $\left(P_{\text {tri }}\right)$ and the patient's PT value. As shown in Figure 10, the trigger sensitivity of the normal trigger is $2 \mathrm{cmH}_{2} \mathrm{O}$. As the trigger sensitivity increases, the value of $P_{\text {tri }}$ decreases to $1 \mathrm{cmH}_{2} \mathrm{O}$ and the patient's PT value is smaller. Therefore, the value of $P_{\mathrm{S}}$ needs

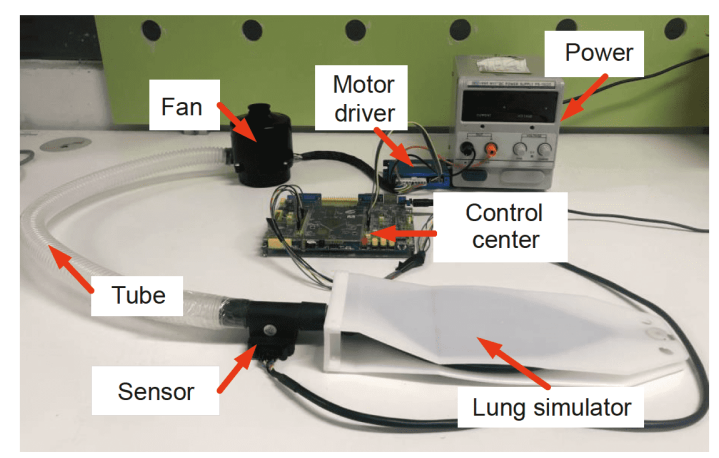

Figure 9 (Color online) Experimental prototype of the ventilator. This prototype is controlled by a MCU and can work offline.

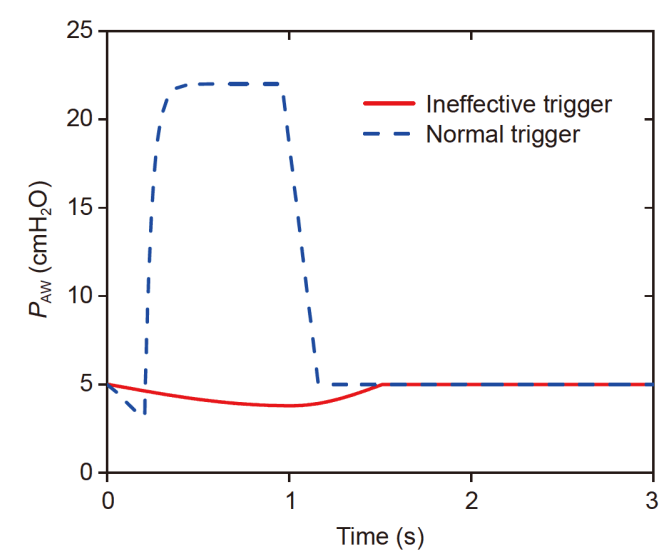

Figure 10 (Color online) Ineffective triggering during pulmonary rehabilitation. The solid line represents the pressure in airway under ineffective triggering, and the dotted line represents the pressure in airway under normal triggering. to be promoted properly, so as to provide more support to the patient's inspiratory.

\subsection{Asynchrony phenomenon of double triggering}

As the patient's spontaneous breathing intensity increases, the degree of pressure support of the ventilator can be gradually reduced. This process aims to train the patient's respiratory ability. The inspiration-to-expiration ratio can be adjusted by the increasing of the switching coefficient $(k)$. The adjustment of the $k$ value can also adapt to the increase of the patient's breathing rate during the pulmonary rehabilitation. If the value of $k$ is set too high, the patient will be forced to switch to the exhalation phase before completing the inhalation phase. At this point, if the negative pressure generated by the patient's breathing effort reaches trigger sensitivity, it will trigger the second pressure support of the ventilator, causing a double triggering asynchrony.

In addition, when the patient's willingness to breathe spontaneously increases suddenly, the peak volumetric flow is higher than the normal level. Then it is possible to reach the switching condition prematurely, resulting in insufficient tidal volume, thus causing the asynchrony phenomenon of double triggering.

To simulate the patient's effort during double triggering phenomenon, the trigger sensitivity is adjusted differently in a breathing cycle. As shown in Figure 11, the initial trigger sensitivity is $2 \mathrm{cmH}_{2} \mathrm{O}$, and then the trigger sensitivity increases to $3.5 \mathrm{cmH}_{2} \mathrm{O}$. When the double triggering occurs, the patient's effort can also trigger the ventilator, even though the trigger sensitivity has been promoted dramatically. It proves that the double triggering is often accompanied by a sudden increase of patient's breathing willingness.

The double triggering asynchrony can be determined by measuring the interval time between two adjacent triggers. If

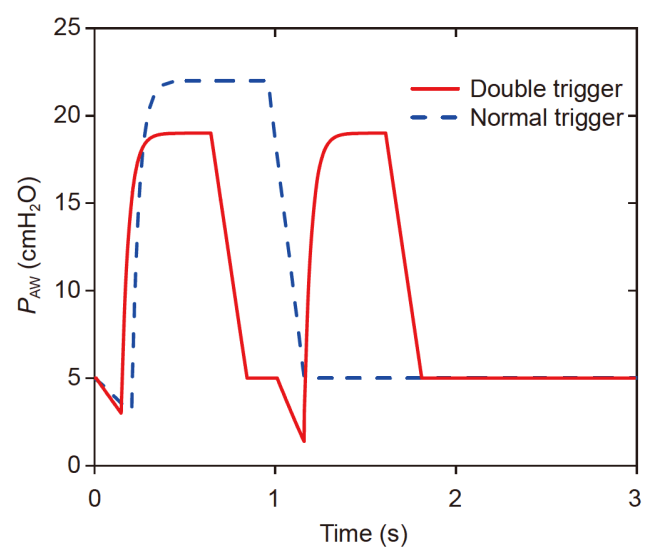

Figure 11 (Color online) Double triggering during pulmonary rehabilitation. The solid line represents the pressure in airway under double triggering, and the dotted line represents the pressure in airway under normal triggering. 
the interval is less than half of the inhalation phase, it can usually be regarded as a double triggering. When the double triggering asynchrony occurs, the value of $k$ needs to be lowered in time to increase the inspiratory time and ensure sufficient tidal volume for the patient.

\subsection{Asynchrony phenomenon of delayed switching}

In the process of pulmonary rehabilitation, when the patient recovers from controlled ventilation without spontaneous breathing to assisted ventilation with triggering, the ventilator needs to adjust the inspiratory-to-expiratory ratio as the frequency of the patient's spontaneous breathing frequency increases. Patients may experience switching asynchrony when the ratio is not adjusted properly. If the value of $k$ is set too low, the patient will still be in the inhalation phase when he wants to exhale, causing the delayed switching asynchrony.

As shown in Figure 12, the solid curves represent the pressure curve and the flow curve in the ventilation cycle with delayed switching. The part circled by the dotted line indicates the characteristic of this phenomenon, that is, the sudden increase in pressure at the end of the plateau pressure and the corresponding scoop-shaped depression of the flow waveform. Compared with the curve of the normal swich, which is represented by the dotted line, the curve of delayed switch has a significant lag in the switching time. The delayed switching asynchrony could be determined by measuring these characteristics.

When the delayed switching occurs, the value of $k$ should be appropriately reduced in time to ensure a proper inhalation-to-exhalation ratio. Ensuring the normal switching between the inhalation phase and the exhalation phase is an important part of the mechanical ventilation strategy for pulmonary rehabilitation.

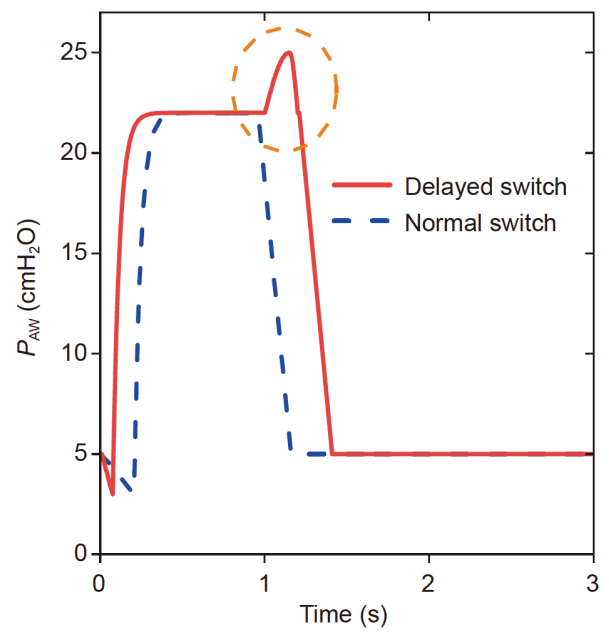

\section{Conclusions}

Pulmonary rehabilitation is essential during the process of mechanical ventilation. And when the patient weans from the ventilator, pulmonary rehabilitation is helpful to train the spontaneous breathing ability. This operation can effectively avoid the weakness of the respiratory muscles during the long-term mechanical ventilation [42]. In this research, the mechanical ventilation strategies for pulmonary rehabilitation are studied based on the patient-ventilator interaction. Firstly, a pneumatic model of patient-ventilation interaction is proposed to simulate the work of the mechanical ventilation system. Based on the pneumatic model, the mathematical relationship between the patient's respiratory system and the ventilator can be established. And the equations of state for pressure, volumetric flow and tidal volume are obtained in this article. An experimental prototype of the ventilator is established to verify the correctness of the pneumatic model and the effectiveness of the ventilation strategy.

In this article, a novel fuzzy control method of the ventilator's support pressure in PSV mode is proposed for the first time. The fuzzy sets of the trigger sensitivity, the value of PT, and the plateau pressure are respectively established. The fuzzy rules are determined according to the clinical experience. Based on the fuzzy rules, the value of plateau pressure that reflects the degree of the pressure support could be obtained by the value of trigger pressure and PT. Among them, PT is an integral quantity that can reflect the intensity of the patient's inhalation effort.

In addition, according to the patient's respiratory mechanics characteristics, each breathing cycle is divided into four phases, namely, (1) triggering phase, (2) inhalation phase, (3) switching phase, (4) exhalation phase. The corresponding ventilation strategy for pulmonary rehabilitation

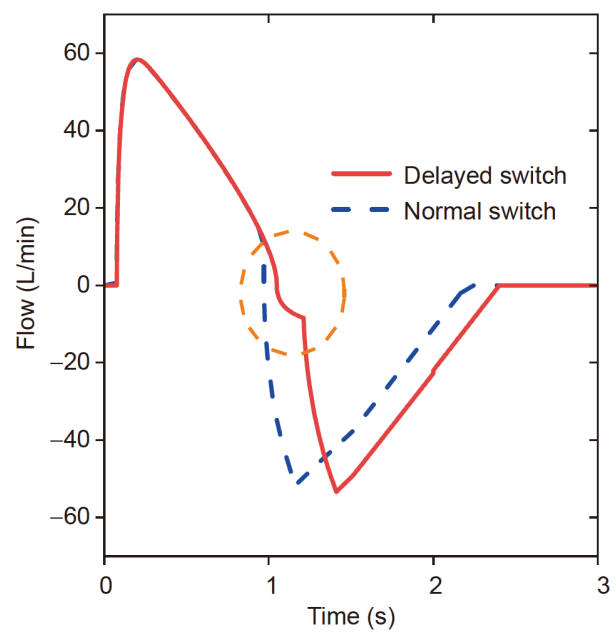

Figure 12 (Color online) Delayed switching during pulmonary rehabilitation. The solid line represents the pressure in airway and the flow under delayed switching, and the dotted line represents the pressure in airway and the flow under normal switching. 
is proposed based on the interaction between the patient and the ventilator at each phase. At last, by analyzing the patientventilator asynchrony phenomena during the pulmonary rehabilitation process, the adjustment method of mechanical ventilation strategy is proposed and discussed in detail.

The results of this research are of great help to the clinical operation in mechanical ventilation. During the process of pulmonary rehabilitation, the patient's ability of spontaneous breathing can be exercised by adjusting the control parameters of the ventilator. It can help the patient get rid of the dependence on the ventilator effectively, so as to complete the weaning more efficiently. It is also helpful for the reduction of the ventilator complications. Besides, this article provides a theoretical basis for the future research on intelligent control methods of the ventilator.

This work was supported by the National Natural Science Foundation of China (Grant No. 52005015), the China Postdoctoral Science Foundation (Grant No. 2019M660391), the Open Foundation of the State Key Laboratory of Fluid Power and Mechatronic Systems (Grant No. GZKF201920), the Outstanding Young Scientists in Beijing (Grant No. BJJWZYJH01201910006021), the National Key Research and Development Project (Grant No. 2019YFC0121700), and the Clinical research support project of PLA General Hospital (Grant Nos. 2019-XXJSYX-13 and 2019XXMBD-013).

1 Ambrosino N, Gabbrielli L. The difficult-to-wean patient. Expert Rev Respiratory Med, 2010, 4: 685-692

2 Wunsch H, Wagner J, Herlim M, et al. ICU occupancy and mechanical ventilator use in the United States. Critical Care Medicine, 2013, 41: A1579

3 Afonso A S M, Verhamme K M C, Sturkenboom M C J M, et al. COPD in the general population: Prevalence, incidence and survival. Respiratory Med, 2011, 105: 1872-1884

4 Cruz A A. Global surveillance, prevention and control of chronic respiratory diseases: A comprehensive approach. World Health Organization, 2007

5 Rabe K F, Hurd S, Anzueto A, et al. Global strategy for the diagnosis, management, and prevention of chronic obstructive pulmonary disease. Am J Respir Crit Care Med, 2007, 176: 532-555

6 Ñamendys-Silva S A. Respiratory support for patients with COVID19 infection. Lancet Respiratory Med, 2020, 8: e18

7 Griffiths R D, Hall J B. Intensive care unit-acquired weakness. Critical Care Med, 2010, 38: 779-787

8 Kress J P, Hall J B. ICU-acquired weakness and recovery from critical illness. N Engl J Med, 2014, 370: 1626-1635

9 Chang A T, Boots R J, Brown M G, et al. Reduced inspiratory muscle endurance following successful weaning from prolonged mechanical ventilation. Chest, 2005, 128: 553-559

10 Petrof B J, Jaber S, Matecki S. Ventilator-induced diaphragmatic dysfunction. Curr Opin Critical Care, 2010, 16: 19-25

11 Boles J M, Bion J, Connors A, et al. Weaning from mechanical ventilation. Eur Respiratory J, 2007, 29: 1033-1056

12 Choi J Y, Tasota F J, Hoffman L A. Mobility interventions to improve outcomes in patients undergoing prolonged mechanical ventilation: A review of the literature. Biol Res Nursing, 2008, 10: 21-33

13 Baptistella A R, Sarmento F J, da Silva K R, et al. Predictive factors of weaning from mechanical ventilation and extubation outcome: A systematic review. J Critical Care, 2018, 48: 56-62

14 Epstein S K. Weaning from ventilatory support. Curr Opin Critical Care, 2009, 15: 36-43

15 Esteban A, Alía I, Tobin M J, et al. Effect of spontaneous breathing trial duration on outcome of attempts to discontinue mechanical ventilation. Am J Respir Crit Care Med, 1999, 159: 512-518

16 Gosselink R, Bott J, Johnson M, et al. Physiotherapy for adult patients with critical illness: Recommendations of the European respiratory society and european society of intensive care medicine task force on physiotherapy for critically ill patients. Intensive Care Med, 2008, 34 : 1188-1199

17 Bissett B, Leditschke I A, Neeman T, et al. Weaned but weary: One third of adult intensive care patients mechanically ventilated for 7 days or more have impaired inspiratory muscle endurance after successful weaning. Heart Lung, 2015, 44: 15-20

18 Schweickert W D, Gehlbach B K, Pohlman A S, et al. Daily interruption of sedative infusions and complications of critical illness in mechanically ventilated patients. Critical Care Med, 2004, 32: 12721276

19 Moodie L H, Reeve J C, Vermeulen N, et al. Inspiratory muscle training to facilitate weaning from mechanical ventilation: Protocol for a systematic review. BMC Res Notes, 2011, 4: 283

20 Moodie L, Reeve J, Elkins M. Inspiratory muscle training increases inspiratory muscle strength in patients weaning from mechanical ventilation: A systematic review. J PhysioTher, 2011, 57: 213221

21 Elkins M, Dentice R. Inspiratory muscle training facilitates weaning from mechanical ventilation among patients in the intensive care unit: a systematic review. J PhysioTher, 2015, 61: 125-134

22 Martin A D, Smith B K, Davenport P D, et al. Inspiratory muscle strength training improves weaning outcome in failure to wean patients: A randomized trial. Critical Care, 2011, 15: 1-12

23 Bonnevie T, Villiot-Danger J C, Gravier F E, et al. Inspiratory muscle training is used in some intensive care units, but many training methods have uncertain efficacy: A survey of French physiotherapists. J PhysioTher, 2015, 61: 204-209

24 Elbouhy M S, AbdelHalim H A, Hashem A M A. Effect of respiratory muscles training in weaning of mechanically ventilated COPD patients. Egyptian J Chest Dis Tuberculosis, 2014, 63: 679-687

25 Huang C H, Yang G G, Wu Y T, et al. Comparison of inspiratory muscle strength training effects between older subjects with and without chronic obstructive pulmonary disease. J Formosan Med Association, 2011, 110: 518-526

26 Prasad N, Cheng L F, Chivers C, et al. A reinforcement learning approach to weaning of mechanical ventilation in intensive care units. arXiv: 1704.06300

27 de Souza L C, Guimarães F S, Lugon J R. Evaluation of a new index of mechanical ventilation weaning. J Intensive Care Med, 2015, 30: $37-43$

28 Jordan J, Rose L, Dainty K N, et al. Factors that impact on the use of mechanical ventilation weaning protocols in critically ill adults and children: A qualitative evidence-synthesis. Cochrane Database Syst Revi, 2016, 10: CD011812

29 Rose L, Fowler R A, Fan E, et al. Prolonged mechanical ventilation in Canadian intensive care units: a national survey. J Critical Care, 2015, 30: $25-31$

30 Hao L, Shi Y, Cai M, et al. Dynamic characteristics of a mechanical ventilation system with spontaneous breathing. IEEE Access, 2019, 7: 172847

31 Shi Y, Niu J, Cai M, et al. Dimensionless study on dynamics of pressure controlled mechanical ventilation system. J Mech Sci Technol, 2015, 29: 431-439

32 Shi Y, Wang Y, Cai M, et al. An aviation oxygen supply system based on a mechanical ventilation model. Chin J Aeronautics, 2018, 31: 197-204

33 Niu J L, Shi Y, Cao Z X, et al. Study on air flow dynamic characteristic of mechanical ventilation of a lung simulator. Sci China Tech Sci, 2017, 60: 243-250

34 Ward M E, Eidelman D, Stubbing D G, et al. Respiratory sensation and pattern of respiratory muscle activation during diaphragm fatigue. J Appl Physiol, 1988, 65: 2181-2189 
35 Jodat R W, Horgan J D, Lange R L. Simulation of respiratory mechanics. Biophys J, 1966, 6: 773-785

36 Mecklenburgh J S, Al-Obaidi T A A, Mapleson W W. A model lung with direct representation of respiratory muscle activity. British $\mathrm{J}$ Anaesthesia, 1992, 68: 603-612

37 Ren S, Cai M, Shi Y, et al. Influence of bronchial diameter change on the airflow dynamics based on a pressure-controlled ventilation system. Int J Numer Meth Biomed Engng, 2018, 34: e2929

38 Ren S, Li W, Wang L, et al. Numerical analysis of airway mucus clearance effectiveness using assisted coughing techniques. Scientific reports, 2020, 10: 1-10
39 Ren S, Niu J, Luo Z, et al. Cough expired volume and cough peak flow rate estimation based on GA-BP method. Complexity, 2020, doi: $10.1155 / 2020 / 9036369$

40 Ren S, Shi Y, Cai M, et al. ANSYS-MATLAB co-simulation of mucus flow distribution and clearance effectiveness of a new simulated cough device. Int J Numer Meth Biomed Engng, 2018, 34: e2978

41 Ren S, Shi Y, Cai M, et al. Influence of airway secretion on airflow dynamics of mechanical ventilated respiratory system. IEEE/ACM Trans Comput Biol Bioinf, 2017, 15: 1660-1668

42 Stawicki S P. Mechanical ventilation: weaning and extubation. Int J Acad Med, 2017, 3: 67 